\title{
Zum Kulturbereich der syrjänischen Kerbkalender
}

Über die Zeitrechnung der finnisch-ugrischen Völker gibt es eine Reihe Untersuchungen, die teils von dem darauf bezüglichen alten Wortbestand teils vom Brauchtum ansgehen. ${ }^{1}$ Einen Beitrag dazu liefern wenigstens für das ausgehende Mittelalter und den Beginn der Neuzeit als gegenständliches Studienobjekt die Runen- bzw. Kerbkalender. Sie sind gleichsam ein Bilderbuch der Volkskultur. Man braucht nur einmal einen Runenkalender aus West- oder Mittelfinnland und einen Kerbkalender aus Karelien² nebeneinander zu halten, um in aller Deutlichkeit die Lnterschiede zweier Kulturbereiche zu sehen - in der Art der Jahresrechumug, der Fest- und Gedenktage, der dafür geformten Zeichen.

Es ist eine bekannte Tatsache, dass unter den verwandten Völkern die Syrjänen besonders eigenartige Kerbkalender besitzen, deren rätselhafte Zeichen schon im vorigen Jahrhundert vor allem syrjänische Forscher zu Lntersuchungen angeregt haben. Weniger bekannt und für Europa eine seltene Erscheinung ist, dass syrjänische Kerbkalender noch bis in das

1 Es sei nur an einige der bekanntesten erinnert wie Y. H. ToIvox Ex, "Vuoden varrelta" in Sana sanasta kaksi parhaasta, Marti Räsänex, "Tscheremissische zeit- und massbestimmungen" MSFOu LII, KustaA Vilkuna, "Wochenrechnung und Teilung des Jahres in zwei oder vier Teile" in Nr. XXXIV, 1 dieser Zeitschrift.

2 Den interessanten Vergleich hat der ausgezeichnete Fachmann auf diesem Gebiet A. HırsJärvı in dem Artikel Länsi ja itä kalenterisauvoissamme in Muinaista ja vanhaa Itä-Karjalaa (als Manuskr. gedruckt) ausgeführt. Herrn lic. phil. Hirsjärvi habe ich viele Ratschläge und Literaturhinweise zu verdanken, wofür ich ihm gleich zu Beginn dieser Arbeit meinen herzlichsten Dank aussprechen möchte. 
letzte Jahrzehnt hinein an vereinzelten abgelegenen ()rt'n bei Jägern in Gebrauch waren. ${ }^{1}$

Die philologischen Fragen, die an die syrjänischen Kerbkalender geknüpft wurden wie der Zusammenhang mit der eigenartigen "permischen Schrift" und mit einem vermuteten zu Grunde liegenden "heidnischen Kalender", sind in der Literatur zwar immer wieder erwähnt, aber noch nicht einer neueren gründlichen Lntersuchung unterworfen worden. Im Folgenden möchte ich mich keineswegs anheischig machen, diese Fragen lösen oder auch nur in die inneren Bezirke der syrjänischen Kultur eindringen $\mathrm{zu}$ wollen. Aber soweit es mir als "Aussenstehendem» möglich ist, möchte ich einige kulturelle Gesichtspunkte, die von allgemeinem Interesse sind, an diesen sichtbirren Zeugnissen einer bestimmten Zeitrechnung, eines alten Brauchtums und ron Ausdrucksformen eines eigenartigen Stils aufzeigen. Vielleicht klingt das zu prunkhaft für einen so einfachen Gegenstand. Aber nähere Betrachtung zeigt, dass verschiedenartigste Elemente sich in ihm vereinigen, die ihrerseits verschiedenen Kulturbereichen zuzuweisen sind. Wir müssen uns also zuerst die syrjänischen Kerbkalender ansehen. Mit der folgenden Darstellung möchte ich die Haupttypen der in Frage stehenden Kalender in einer kurzen I'bersicht beschreiben, weil ich in der Literatur immer nur einzelne Stücke isoliert behandelt gefunden habe. Diese Beschreibung erhebt aber nicht den Anspruch auf fachmännische ethnographische Behandlung des Stoffes. Das muss ich den wenigen Fachleuton, die es auf diesem Gebiet gibt, überlassen. Deshalb nehme ich mir auch die Frriheit, den Haupttypen, wie ich sie verstehe, einen zum Zweck der kürzeren Ausdrucksweise gewählten Jamen zı geben, der also nur als Stichwort, nicht als Fachwort zu gelten hat.

1 Während meines Studienaufenthaltes in Syktyvkar Ende 1961 erklärte mir der Kustos des dortigen Museums, Herr A. M. Ru Bcov, dass er noch i. J. 1958 von dem Syrjänen Alexander Bažukov aus dem Dorf Ust-Ilyt's an der Pečora einen Kerbkalender erhalten habe. 
Typender Kerbkalender

1. De r Kerbholzty p. In seiner "Reise nach dem Nordosten des europäischen Russlands»" ${ }^{1}$ berichtet Alexander G. Schrenk von einem Kalender, den er in der Grossland-Tundra bei dort zeltenden Syrjänen gesehen habe: "Ein vierseitiges Stäbchen, auf dessen zwei gegenüberliegenden Seiten die einzelnen Tage durch Einkerbungen bezeichnet werden, und zwar die gewöhnlichen Wochentage durch einfache Querkerbe, die Sonntage durch Kreuze; Festtage, die auf Wochentage fallen, durch eine in den Querkerb eingebohrte Vertiefung, mit Sonntagen zusammenfallende Festtage durch eine ebenso in das Kreuz eingebohrte Vertiefung, und diese Zeichen schräg gelegt - Fastenzeit». In diesem Stäbchen fand Schrenk die Erklärung für die erstaunliche Tatsache, dass die Syrjänen "hier in der Wildnis ihre Zeitrechnung nicht verloren und die Kirchenfeste, die auf ein bestimmtes Datum fallen, so genau beobachteten.» Er คrfuhr auch, dass dieser Kalender von »Bücherleuten» (Knižniki) in den Dörfern verfertigt wird. Merkwürdig ist seine Erklärung über die Kennzeichnung der bereits vergangenen Tage und Wochen, die in der Weise geschehe, dass die betr. Zeichen weggeschnitten würden. "Wenn sich dann das ganze Kalenderstäbchen, allmählig kürzer werdend, seinem Ende nähert, so macht sich auch der Nomade bereit die Tundra zu verlassen, die er zeitweilig bewohnte, um den Winter in den heimathlichen Dörfern zuzubringen, wo er des Kalenders nicht bedarf.» Dieses cigenartige "Kürzerschneiden" des Stäbchens im Bericht Schrenks beruht offensichtlich auf einem Missverständnis, da mit den ersten verflossenen Wochen gleichzeitig die auf der gegenüberliegenden Seite des Stäbchens eingekerbten Zeichen der späteren Wochen und Monate weggeschnitten worden wären. Den richtigen Sachverhalt erkennen wir aus einer anderen, etwas später veröffentlichten Beschreibung eines gleichartigen Kalenderstäbchens mit Abbildung, ${ }^{2}$ wo zu sehen ist, dass für die letzten Tage des Juli (oben rechts) ein Schnitt längs der Kante des

\footnotetext{
1 1. Teil, lorpat 1848 , S. $330-331$.

2 П. И. Кузнецовъ, Природа и жители востичнаго склона сенернаро эрала. Ианғстія И.Р.Г.Обц. XX111,6, 1887, p. 717- 749- Tafel.
} 
Stäbchens gemacht ist. Zwei derartige Kalenderstäbe von 74,5 und $62,5 \mathrm{~cm}$ Iänge, beide aus dem syrjänischen Gebiet (davon eines von östlich des Ural wohnenden syrjänen) erhalten, besitzt das Lngarische Nationalmus' InII. Aus der Beschreibung

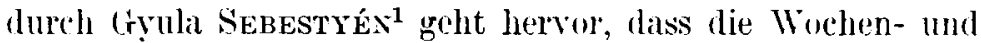
Sonntage durch die gleichen Zeichen (gerade Striche und schrär liegende Kreuze) bezeichnet sind; von seitlichen Einschnitten zur Kennzeichnung der verflossenen Zeit ist aber nichts zu sehen. Während das längere Kalenderstäbchen in der fortlaufenden Reilıe der Kerbzeichen auf zwei gegenüberliegenden Seiten für die Tage des Jahres keinerlei Monatsteilung zeigt - auf den freien Flächen sind statt dessen die Anfangsbuchstaben der russ. Monatsnamen mit Bleistift an den entsprechenden Stellen (vom Sammler?) vermerkt - sind auf jeder Seite des kürzeren Stäbchens jeweils drei Monate durch Abstände erkenntlich. IDis Festtage sind wie in den rorher genannten Kalendern durch punktartige Vertiefungen gekennzeichnet. Wegen der festliegenden Reihe der Sonn- und Wochentage ist auch das Jahr, für das diese Kalender gemacht sind, bestimmbar. Es beginnt in beiden von Sebestyén beschriebenen mit einem Sonntag (1. Januar) und liess sich als d.J. 1889 berechnen. Merkwürdigerweise erwähnen die genannten Verfasser nur diesen Kalendortyp, so dass der Eindruck entsteht, als sei dieser dor einzigr oder vorherrschende bei den Syrjänen. ${ }^{2}$

Vgl. I . T. Sirelıs, Suomen kansanomaista kulttuuria II, I lelsingissä 1921, S. 535 , Abb. 708 .

1 Rovás és rovásirás, Budapest 1909, S. 94 u. 95, wo auf der Abb. 56 beide Kalenderstäbe zu sehen sind.

2 Vgl. Carles Raвот, A travers Ia Russie Boréale, Paris 1894; L. RütıMEYER, Ur-Ethnographie der Schweiz, Basel 1924, wo S. 35-36 ein tschuwassischer Kalenderstab (mit derselben Art der Wuchen- und Feiertagsbezeichnung wie oben) beschrieben und abgebildet ist mit dem Zusatz: "Sie (diese Kalendarien) werden für ein ganzes oder halbes Jahr angefertigt und sind nach Mitteilung von Prof. Adler (Direktor der Sammlung für Völkerkunde in Kazan) bei den finnischen Völkern beiderseits des Ural sehr verbreitet». Auch Sigfrid Svessson erwähnt in B o n d e n s $\AA$ r, Stockholm 1945, als syrjänischen Kalender nur diesen für ein Jahr brauchbaren Typ. Beachtenswert ist das Verbreitungsgebiet dieses Kalenders. Genau diesen Typ brachte T. Lentisalo als Kerb- 
Er erfreut sich jedoch wohl zu Unrecht dieser Einschätzung, schon deshalb, weil sein Gebrauch auf ein bestimmtes Jahr oder einen Teil des Jahres beschränkt ist. Ausserdem sind die für die Kalenderrechnung gebrauchten Zeichen (vertikale Striche und wie bei einer römischen $\mathrm{X}$ krenzweise übereinandergelegte Striche) die im Zählsystem der Kerbhölzer typischen Zahlzeichen. ${ }^{1}$ Mit den Kerbhölzern rerbindet diesen Kalendertyp auch die erwähnte Praxis des Wegschneidens "ungültig" gowordener Zeichen für die vergangenen Tage. Um ihn von den im Folgenden zu behandelnden syrjänischen Kerbkalenderı zu trennen, möchte ich ihn hier "Kerbholztyp" nennen.

2. D e r J äg e r k a l e n d e r t y p. Auf dem Ersten Archäologenkongress in Moskall 1869 hat P. I. SAvvarrov, ${ }^{2}$ dessen Hauptverdienste auf dem Gebiet der syrjänischen Sprache liegen, einen syrjänischen Kerbkalender beschrieben, dessen geometrische, buchstabenähnliche Zeichen er eingehend untersuchte. Jer Vergleich mit den skandinavischen Runenkalendern und einige Übereinstimmungen mit Schriftzeichen des im Altsyrjänischen gebrauchten "permischen Alphabets" führten ihn zu dem Schluss, der Apostel der Syrjänen, Bischof Stephan, habe diese Zeichen als Grundlage zur Schaffung der permischen Schrift benutzt, dabei den schon existierenden wheidnischen Kalender» der Syrjänen übernommen und dem christlichen Kalender angepasst. Die Auffassung Savvaitovs von dem hohen Alter und heidnischen Ursprung dieses Kerbkalenders wirkt noch bis in die Gegenwart fort. In ihrem ausgezeichneten ethnographischen Werk über die Syrjänen ${ }^{3}$ zitiert V. N. BeLIcen

kalender der Samojeden aus Obdorsk, wo er anscheinend auf Bestellung angefertigt und ihm verkauft wurde, nach Helsinki. Er befindet sich im Besitz des Finnischen Nationalmuseums.

1 Vgl. ausser Senestyén und Rítineyer auch M. Blch, Die Wotjäken, Acta Soc. Scient. Fenn. T. XII. Helsinki 1881; В.т. Вл. Богдановъ, Русская бирка, Этнографическое Ооозрьніе СIX-CX. 1916.

2 II. И. Савваитов, О зырннскихъ деревянныхъ календаряхъ и пермской азбукъ, Труды перваго археологическаго съ ъзда въ Москв 1869, II. S. 408-416 (Abb. S. 408).

3 В. И. Белицер, Очерки по әтнографии народов номи $\mathrm{XIX}-$ начало XX в. Москва 1958, S. $71-72$ und. Anm. 127. 
Savvaitov und spricht ebenfalls von wheidnischen Kalendern". Wie schon eingangs gesagt, nötigt uns gerade diese Auffassung zu näherer Untersuchung der syrjänischen Kerbkalender.

Der von Savvaitov beschriebene Kalender ist ein sechskantiges, von der Mitte nach beiden Enden konisch zulaufendes prismaförmiges Holzstäbchen von ca. $18 \mathrm{~cm}$ Länge. In der Mitte befindet sich eine rundherumgehende Vertiefung, durch die die 6 Kanten in 2 Hälften geteilt werden. Dadurch ist eine ausserordentlich klare Einteilung in die 12 Monate des Jahres gereben. Auf jeder Kantenhälfte ist die Zahl der entsprechenden IIonatstage (31 bzw. 30 u.i. Februar 28) durch Einkerbungen in gleichen Abständen vermerkt, so dass das ganze Stäbchen gezähnt anssieht. Wocheneinteilung oder Bezeichnung der Sonntage ist nicht zu sehen. Dagegen sind auf den Flächen des Stäbchens zu einzelnen Monatsdaten, auf die bestimmte Fest- oder ( redenktage fallen, bei der Kerbstelle geometrische Zeichen und Figuren eingegraben: die Kennzeichen der unbeweglichen Feste. Savraitov stellt sie mit den entsprechenden Angaben des kirchlichen orthodoxen Kalenders zusammen. Wegen dieser Zeichen, die offensichtlich das wichtigste mnemotechnische Mittel des Kalenders sind, wird er vom Volk »Pas» genannt, womit man im Syrjänischen Eigentums- bzw. Familienzeichen meint.

Diese Art Kalender muss ziemlich weit verbreitet gewes'n sein, denn noch in einer ethnographischen Schilderung des Syrjänenlandes durch einen Reisenden ${ }^{1}$ vom Jahre 1928 finden wir cine anschauliche Schilderung davon. Er sah iln in Gebrauch bei einem șrjänischen Jäger in dessen Waldhütte. Aus der Beschreibung erfahren wir als nel, dass die Sonntage des betr. Jahres dadurch bezeichnet waren, dass die entsprechenden Kerbstellen mit Wachs ausgefüllt wurden.

Als ich im Dezember 1961 bei meinem Studienaufenthalt in Syktyvkar das Republikanische Heimatkundemuseum öfters besuchte, bemerkte ich in einer Vitrine unter Ausrüstungsgegenständen der Jäger zwei Kerbkalender der von Savvaitov beschriebenen Art, die als "Jägerkalender" (und auch "Pasjas»)

1 С. Сергель, В Зарянском крае. Москва-Ленинград 1928, \$. 49 (mit Zeichnung). 
bezeichnet waren. ${ }^{1}$ Auf meine Frage erklärte mir der Kustos, Herr A. M. Rubcov, dass das Museum eine ganze Reihe solcher Kalender besitze. İ̉ber den von ihm selbst noch i.J. 1958 bei einem Syrjänen an der Pečora angetroffenen und mitgebrachteı Kalender habe ich oben schon berichtet. Ich konnte mehrere Exemplare studieren und mir Aufzeichnungen machen, die ich im Folgenden verwerte. Der Direktor des Museums, Herr M. Kalinin - das Museum steht in enger Zusammenarbeit mit der Filiale der Akademie der Wissenschaften - hat mir ausserdem freundlicherweise erstklassige vollständige Fotokopien zweier Kerbkalender nach Helsinki nachgesandt, die hier (Abb. 1.) zur Veröffentlichung gelangen. Sie sind der sichtbare Beweis für das grosse Entgegenkommen der Museumsbeamten und Wissenschaftler in Syktyvkar, denen ich an dieser Stelle meinen herzlichen Dank für die wertvolle Hilfe ausspreche.

Wie aus der obigen Beschreibung und den Bildern zu ersehen ist, beruht die Zeitrechnung auf dem Julianischen Kalender, wie er von der orthodoxen Kirche in Russland gebraucht wurde. Ein wichtiger Punkt ist dabei zu beachten: der Jahresanfang liegt auf dem 1. März, wie es bis ins 14. Jahrhundert Brauch war, selten auf dem 1. September, ${ }^{2}$ der danach der offizielle J ahresanfang war, und nie auf dem 1. Januar, der seit 1700 unter Peter dem Grossen nach westlichem Vorbild zum Jahresanfang wurde. Auf diesen Umstand gründen Savvaitov und andere Forscher, wie noch zu zeigen sein wird, ihre Datierung dieses Kalenders ins 14. Jh.

Ausser dem Jahresanfang ist für die Kalenderrechnung besonders charakteristisch die Einteilung des Jahres in Monate und nicht in Wochen, für die kein Interesse besteht, da nach den Festtagen gerechnet wird. Der Sonntag war für den in den Wäldern lebenden Jäger nicht von Belang. Den Namen Jägerkalender verdient dieses äusserst leichte und handliche Holzstäbchen, weil vor allem die Jäger es in ihrer Kleidung mühelos

1 Die Abb. findet sich in meinem Bericht in Virittäjä 1962, S. 65 (Abb. 3).

2 Nach U. T. Sirelius, Suomen kansanomaista kulttuuria II, llelsingissä 1921, S. 540 kommt auch der Jahresanfang am 1. September vor. 


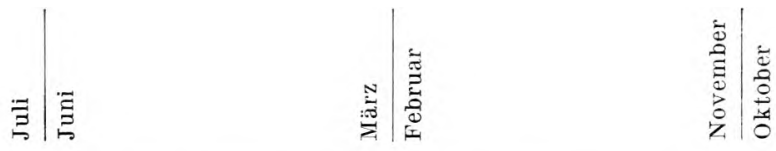

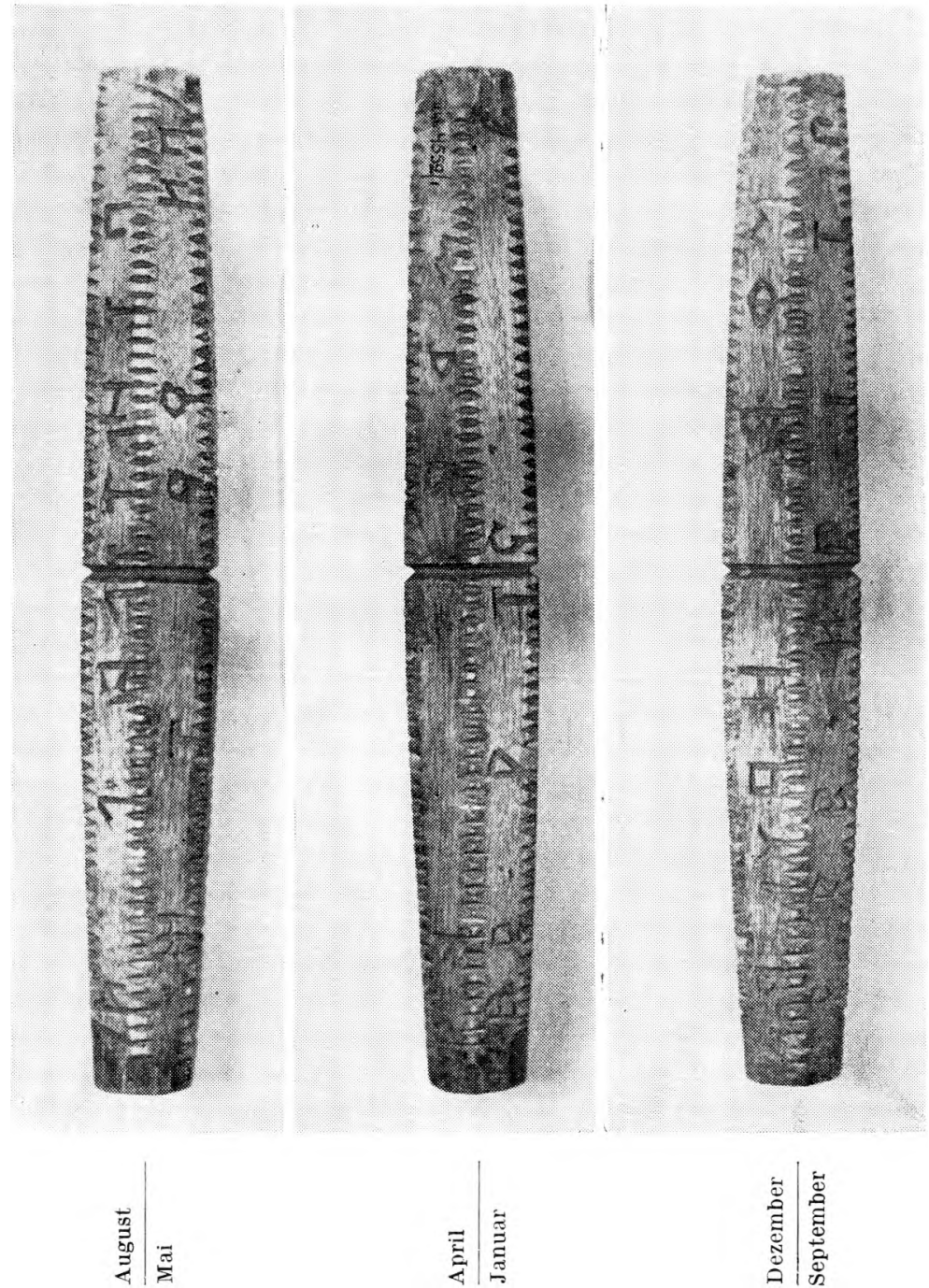

Abb. 1 a,b. Jägerkalender (Pas) der Syrjänen. Republikanisches Heimatkundemuseum in Syktyvkar, Komi ASSR. Foto: Museumsdirektor M. Kalinin. 


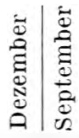

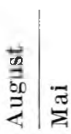

$$
\text { 灵| }
$$
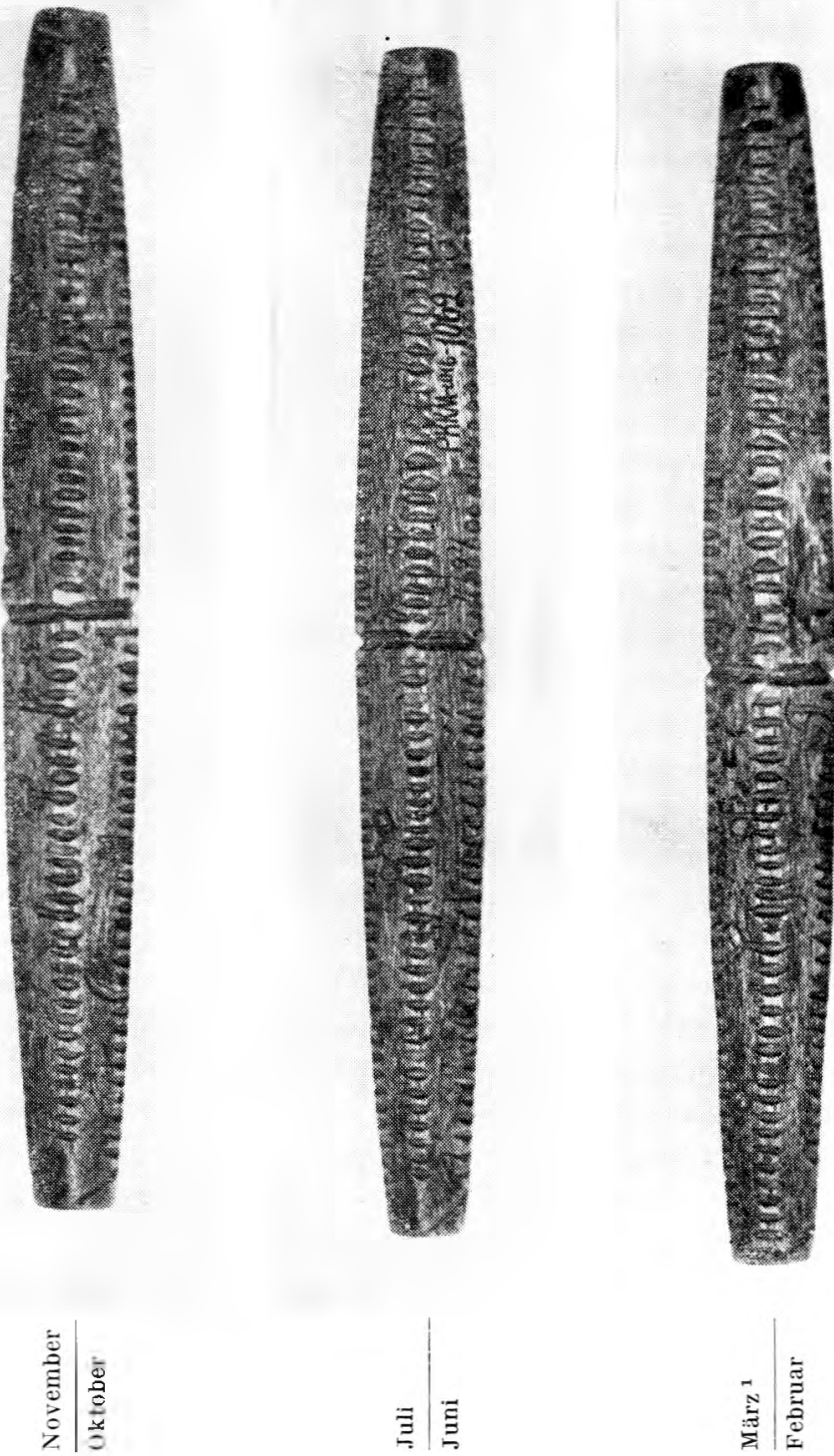

$$
\text { 荧| }
$$

1 Die Einkerbung am linken Ende der Kante zeigt den Anfang der Kalenderrechnung, die mit März beginnt und auf dieser Kante nach rechts verläuft, wo am Ende ein Schrägstrich zur oberen Kante führt und die Zählung von rechts (Mai) nach links (Juni) geht, von dort zum Juli usw., mit jeder auf den Zählenden zu gedrehten kante die Richtung wechseind. 
auf ihre Wanderungen mitnehmen konnten und weil es seit Ende des 19. Jhs. nur von ihnen gebraucht wurde.

3. D e r B a u e r n k l e n d e r ty p. In dem erwähnten Museum in Syktyvkar bemerkte ich in der Abteilung für alte landwirtschaftliche Geräte noch einen als "Pas-Kalender» bezeichneten Kalenderstab, der in Form und Zeichen von dem Jägerkalender sehr verschieden ist. Er stammt aus dem Dorf Vizinga (Bez. Sysolsk) und war dem Museum von A. I. Peršinal geschenkt worden. Es ist ein vierkantiger, einige Zentimeter breiter und etwas über $1 \mathrm{~m}$ langer Holzstab. Auf zwei Flächen sind die Tage für jeweils 6 Monate durch Kerbeinschnitte zur Kante hin seingetragen»; die Monate selbst sind voneinander durch Abstände und ausserdem durch ein auf diesen befindliches Kerbzeichen $\subset$ deutlich getrennt. Auf den darüberliegenden Flächen sind einzelne Festtage zu bestimmten Monatsdaten durch eingeschnittene lange Striche, an einigen Stellen auch durch andere, sehr einfache geometrische Linien, z.B. / und $/$ vermerkt. ${ }^{1}$ Von dem Vorhandensein eines solchen Kalendertyps bei den Syrjänen habe ich bisher weder etwas gesehen noch in der Literatur erwähnt gefunden. Mir scheint aber, dass es sich um einen recht alten Kalendertyp handelt, der für unsere Untersuchung noch von Wichtigkeit sein kann. Charakteristisch ist für diesen wie für den Jägerkalender die Einteilung des Jahres in Monate und überhaupt das kirchliche orthodoxe: Kalendersystem.

Die Gegenüberstellung des Jäger- und des Bauernkalendertyps zeigt, dass die kurze Prismaform des erstgenannten und seime eigenartige Graphik kulturelle Elemente von besonderer Art darstellen. Diese müssen in engem Zusammenhang mit den Lebensgewohnheiten und den gesellschaftlichen Formen der Waldbewohner stehen, so dass der Kulturbereich, dem sie zugehören, wohl auch dort zu suchen sein wird. Die Übereinstimmung der Kalenderrechnung beider Typen mit dem kirchlichen orthodoxen Kalender deutet hingegen darauf hin, dass der dafür in Frage kommende Kulturbereich ein viel weiterer

1 Meine damaligen Aufzeichnungen waren flüchtig, weil ich noch nicht an das jetzt zu hehandelnde Thema dachte. 
sein muss. Diesem nachzugehen soll unsere erste Aufgabe sein.

Sie wäre verhältnismässig leicht, wenn uns Holzkalender der orthodoxen Russen in der Nachbarschaft der Syrjänen bekannt wären. Das ist leider nicht der Fall. Es ist deshalb verständlich, dass Savvaitov die skandinavischen Runenkalender zum Vergleich heranzog. Dazu verlockte ihn ausser vermeintlichen Ähnlichkeiten der "Buchstaben» des Jägerkalenders mit den Runen auch die seit Olaf Rudbeck herrschende und in dem Wunsch nach einem alten "Germanenerbe" noch vor einigen Jahrzehnten zäln festgehaltene Auffassung von dem heidnischen Ursprung' der Runenkalender. Es ist deshalb am Platz festzustellen, dass die Runenkalender nach dem heutigen Stand der Forschung ${ }^{1}$ im Norden erst nach der Verbreitung des Christentums in allen Volksschichten aufgekommen sind und auf die geschriebenen kirchlichen Kalender zurückgehen. Als Vorgänger der Runenkalender ist ein auf Pergament in Runen geschriebenes Kalendarium von Gotland aus d.J. 1328 anzusehen. Der älteste uns bekannte schwedische Runenstab stammt aus d.J. 1504, der älteste finnische aus 1566. Für die Frage nach dem Ursprung ist vor allem das System der Kalenderrechnung entscheidend. Wir können uns ohne Zweifel der Ansicht A. Hirsjärvis anschliessen, der op.cit. S. 21 sagt: "Da der Runenkalender seiner Struktur nach dem im Mittelalter den Bedürfnissen der Kirche angepassten Julianischen Kalender vollständig entspricht, besteht kein (trund zu dem Versuch, für ihn eine hypothetische heidnische Vorstufe, zu der man doch wohl niemals eine Entsprechung in der Wirklichkeit finden kann, zu rekonstruieren. Die Tatsache, dass das Runenalphabet im Kalender benutzt worden ist, braucht keineswegs auf das Heidentum hinzuweisen; uns sind Runeninschriften auf Denkmälern für Getaufte wie auch auf mit dem eigentlichen kirchlichen Kult verbundenen Gegenständen wie Taufbecken und Kirchenglocken recht gut bekannt.» Die Runenkalender bieten also keine Stütze für die Annahme einer heidnischen Vorstufe der syrjänischen Kerb-

1 Vg]. U.a. S. Svensson, Bondens år. Kalender och märkesdagar, hushållsregler och väderleksmärken. Stockholm 1945, S. 57 u.a.a.O.; A. Hırsjärvı "Riimusauva, suomalaisen almanakan edeltäjä» in: Suomen Almanakan Juhlakirja, Helsinki 1957, S. 21 ff. 
kalender. Auch der Gedanke an irgendeine Zusammengehörigkeit dieser Kalender ist auszuschliessen. (Obwohl die Zeitrechnung in beiden auf dem Julianischen Kalender in christlicher Form beruht, zeigen sich doch im Kalendersystem ganz auffallende Unterschiede. Auf dem Runenstab wiederholt sich, um mit A. Hirsjärvi zu sprechen ${ }^{1}$, "in einer Serie von 365 den Jahrestagen entsprechenden Zeichen die Aufeinanderfolge von sieben Runenbuchstaben 52mal (diese Serie ist auf dem Runenstab gewöhnlich in zwei vder vier 'Teile geteilt; die Monatseinteilung ist ausserordentlich selten)». Der Buchstabe, auf den der crste Sonntag des mit dem 1. Januar beginnenden Jahres fällt, bezeichnet das ganze Jahr hindurch den Sonntag (= der Somntaursbuchstabe des betr. .Jahres). Dadurch steht im Mittelpunkt dieses Kalendersystems die Wochenrechnung und die 'Teilung des Jahres in zwei oder vier Teile?

Die oben dargestellte Teilung des Jahres in Monate auf den syrjänischen Jägerkalendern und dem Bauernkalender aus Vizinga wie auch das gänzliche Fehlen der Wochenrechnung in der Aufeinanderfolge der gleichförmigen Kerben für die Jahrestage (im ganzen ebenfalls 365) bilden einen so deutlichen Unterschied zu den Runenkalendern, dass man für jene ein Kalendersystem annehmen muss, das einem anderen Kulturbereich zuzuweisen ist. Hinzu kommt die Verschiedenheit im Jahresanfang. In dieser Auffassung werden wir durch den Vergleich mit den karelischen Kerbkalendern bestärkt. Zu ihren Hauptmerkmalen gehören nach A. HirsJärvi ${ }^{3}$ : die Monatscinteilung (bei den vierkantigen stäben sind auf jeder Fläche drei, bei den dreikantigen auf jeder Fläche vier, bei den sechskantigen auf jeder Fläche zwei Monate markiert), die innerhalb dieser Einteilung eingeschnittenen Kerben zur Bezrichnung der Monatstage ohne Unterscheidung von Somn-oder Wochentagen, das Fehlen der sog. "Goldenen Zahl" und der Jahresanfang mit dem 1. März (gewöhnlich) oder nit dem 1. September (in einigen

1 op. cit. S. 17. Dort findet sich auch alles Wichtige über die Zeichen für die Festtage und die sog. "Goldene Zahl".

2 Vgl. K. VILKUNA, op.cit. S. 74.

${ }^{3}$ Länsi ja itä kalenterisauvoissamme, S. 242. 
Fällen), aber nie mit dem 1. Januar. Die durch Zeichen vermerkten Feste entsprechen dem kirchlichen orthodoxen Kalender. Es ist auf den ersten Blick zu sehen, dass wir es hier mit demselben Kalendersystem zu tun haben wie bei den erwähnten syrjänischen Kerbkalendern. Unterschiede bestehen hauptsächlich in der klobigen, oft kurzen, aber dicken Form der Kalenderstäbe, in der Bezeichnung des Schalttages, wodurch die Gesamtzahl der Jahrestage 366 beträgt, und in den eindeutig christlich-religiösen Symbolen für die Festtage. In dieser Hinsicht ist die schon von U. T. Sirkuius gemachte Bemerkung ${ }^{1}$ aufschlussreich, dass die karelischen Kalender vor allem in Gebieten vorkommen, wo die Altgläubigen stark vertreten waren. Sirelius neigte zu der Auffassung, dass die Kerbkalender aus Finnland (Kainu) nach Karelien gewandert seien. Von dort aus, so scheint er zu glauben (op.cit. S. 546), seien sie durch die Altgläubigen nach dem Osten, vor allem nach sibirien verbreitet worden. Richten wir also unseren Blick nach Sibirien!

Schon Gottlund hatte sich für einen Kerbkalender, der in Omsk als Museumsstück aufbewahrt war, interessiert und eine Kopie davon (heut im Finnischen Nationalmuseum in Helsinki) beschafft. Dieser Holzkalender gleicht in Grösse, Form, Graphik und Kalenderrechnung dem von Savvaitov beschriebenen Jägerkalender. Er wurde ca. 1854 im Bezirk Omsk erworben, wie Vj. SreznevskiJ in seiner Untersuchung über die Runenkalender ${ }^{2}$ glaubwürdig berichtet. Dieser Forscher hat sich bemüht, das gutzumachen, was er an Savvaitovs Methode tadelt, nämlich, dass dieser nur einen einzigen Kerbkalender untersuchte, ohne Vergleichsmaterial heranzuziehen, wodurch er zu seiner "reichlich kühnen Schlussfolgerung» von der Existenz der syrjänischen Kalender schon in heidnischer Zeit gelangte. ${ }^{3}$

Ausser dem Kerbkalender aus Omsk, den er genau beschreibt und abbildet (op.cit. Tafel IV, Zeichn. 5 und Tafel VI, Zeichn. 1), nennt er noch vier aus Mammutknochen verfertigte Kalen-

1 Siehe seine Abhandlung über die alte finnische Zeitrechnung und die Kalender in: Suomen kansanomaista kulttuuria II, Helsingissä 1921, S. 545 u. 546 .

${ }^{2}$ Съверный ръзной календарь. Санктпетербургъ, 1874, S. 50.

3 ibid. S. 49. 
der, die dem oben beschriebenen Jägerkalendertyp gleichen. ${ }^{1}$ Sie stammen alle aus Sibirien. Einer davon aus der Gegend von Turuchansk, ein anderer direkt aus den Händen der Jakuten: es ist der durch den Naturforscher A.Th. von MiddendorfF ${ }^{2}$ beschriebene und abgebildete Jakuten-Kalender Kardî. Wie aus der Abbildung 2 zu sehen ist, handelt es sich um genau denselben Jägerkalendertyp, den wir bei den Syrjänen kennen gelernt haben. Bis an den Baikal führt uns schliesslich K. D. Logrnovskis. ${ }^{3}$ Er bietet Beschreibung und Bilder zweier Knochenkalender der Burjaten aus der Umgebung von Nertschinsk. (Abb. 3). Sie gleichen, abgesehen von Varianten im Jahresanfang, den bisher besprochenen syrjänischen und sibirischen Jägerkalendern. Wie ist diese auffallende Übereinstimmung und das gewaltige Verbreitungsgebiet, das von der Vyčegda und Pečora westlich des Urals bis zur Lena und zum Baikalsee reicht und bei so verschiedenen Völkerschaften anzutreffen ist, zu erklären?

Bezüglich der sibirischen Kalender sind sowohl von Middendorff wie auch Sreznevskij und Loginovskij der Ansicht, dass sie durch russische Kolonisten nach Sibirien gebracht wurden. Dafür spricht vor allem die Tatsache, dass Kerbkalender auch von russischen Bauern und Jägern in Sibirien gebraucht wurden. Sreznevskij berichtet (op.cit. S. 52 nach Mitteilungen, die er erhielt), dass die russischen Jäger an der Lena hölzerne vierkantige Kerbkalender von ca. $70 \mathrm{~cm}$ Länge auf ihre $1 \frac{1}{2}$ Monate dauernden Bärenjagden mitnahmen (sie trugen sie am Gürtel und hängten sie am Lagerplatz an einen Baum). Es scheint jedoch, dass diese mit den oben erwähnten Kerbholzkalendern gleichzusetzen sind. Sreznevskij glaubt denn auch, dass sie aus den Gouvernements Archangelsk und Olonec nach dem Osten gewandert seien. Man kann sie wohl kaum einer bestimmten Völkerschaft, auch nicht der russischen eindeutig zuschreiben. Klarer liegt die Sache betreffs des Bauernkalenders, den P. A.

1 ibid. S. $51-52$.

2 Reise in den äussersten Norden und Osten Sibiriens, Bd. IV, Theil 2: Die Eingeborenen Sibiriens, St. Petersburg 1875, S. 1541.

3 Живая старина XII, 1. С. Петербургъ, 1902, S. 195 ff. mit 4 Tafeln. 


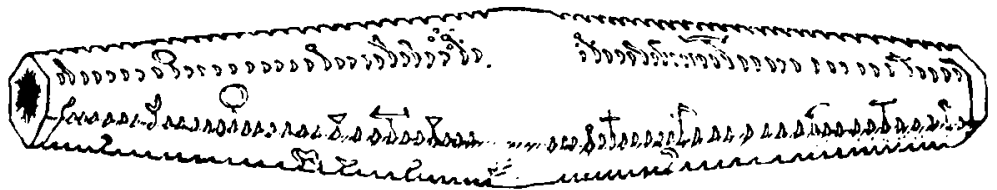

Abb. 2 Jägerkalender der Jakuten. Zeichnung von $\mathbf{K}$. D. Loginovskij in ;нивая старина XII, 1, St. Petersburg 1902, S. 195 ff., Tafel 4. Vgl. den in A.Th. von Middendorffs "Reise in den äussersten Norden und Osten Sibiriens" (Bd. IV, Theil 2, S. 1541) beschriebenen Kalender.

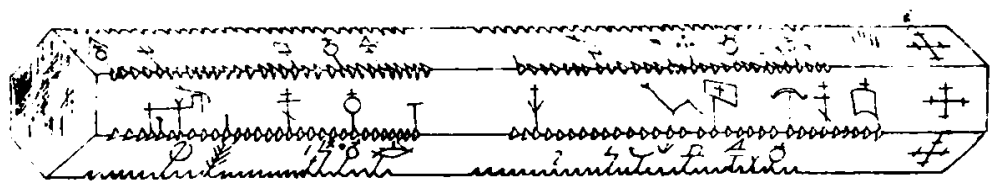

Abh. 3 Kalenderstah der Burjaten am Baikal. Zeichnung von K. D. Loginovskij op.cit. Tafel 3.

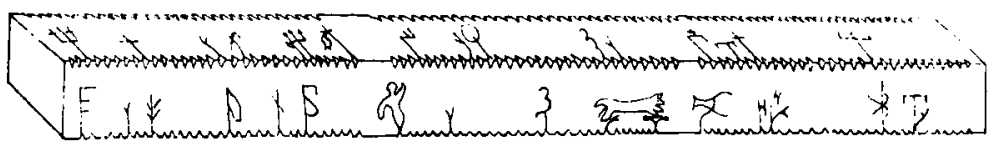

Abb. \& Kalenderstab eines russischen (altgläubigen) Bauern an der Lena. Zeichnung von Loginovskij op.cit. Tafel 1.

Pežemskij auf dem Wege zwischen Jakutsk und Irkutsk bei einem russischen Bauern sah (und abzeichnete, da der Bauer ihn als altes Familienerbe, das an die Kinder weiter gehen sollte, um keincn Preis hergeben wollte). Es war ein vierkantiger ca. $54 \mathrm{~cm}$ langer und $4 \mathrm{~cm}$ breiter Holzstab, dessen Kalenderrechnung mit September als Jahresanfang und christlich-religriösen Zeichen für eine Reihe Feste an die karelischen Kerbkalender der Altgläubigen erinnert. Einige der Zeichen für die (redenktage stimmen jedoch mit denen der sibirischen Jägerkalender überein. Diesem sehr ähnlich ist ein von Loginorskij² veröffentlichter rierkantiger Kerbkalender, der in ten 50er Jahren des 19. Jhs. bei einem russischen Bamern an der Lena

\footnotetext{
1 "Сельскій деревянный календарь" in Москвитанин за $1852 \mathrm{r}$. VII.

${ }^{2}$ op. cit. S. $196 \mathrm{ff}$.
} 
mach dlem (riginal kopirert wurde (Abb. 4). Dir I†bereinstimmungen mit den jakutischen und burjatischen Kalendern sind weitgehend. Der Hauptunterschied besteht darin, dass diese die für die Jägcrkalender charakteristische nach den Enden konisch zulaufende Prismaform haben und kleiner, handlicher sind. Auf Grund dieses Vergleichsmaterials müssen wir wohl der Ansicht beistimmen, dass die sibirischen Kerbkalender sowohl als Batuernkalender- wie als Jägerkalendertỵp von russischen Kolonisten nach dem Osten gebracht wurden. Das passt auch zu der Tatsache, dass seit der Eroberung Sibiriens durch Jermak und seine Kosaken die sog. "Promyschlenniki" ("Gewerbetreibenden"), deren Hauptgewerbe die Jagd war, weit in das Innere Sibiriens eindrangen. Es besteht wohl kein Grund, die Richtung dieser Kolonisatoren aus dem Norden herzuleiten. Dass Altgläubige ausgerechnet aus Karelien den Kerbkilendern eine derartige Verbreitung nach Ostsibirien verschafft haben, ist kaum wahrscheinlich. Auch das Gouvernement Archangelsk kann man nicht als das Ausgangsgebiet ansehen. Sowohl das Gebiet der Pečora wie der Lena und des Baikalsees müssen wir wohl als Peripherie betrachten, an der sich Kerbkalender erhalten haben, die von einem gemeinsamen Zentrum ausgegangen sind. Wie das übereinstimmende Kalendersystem zeigt, muss dieses Zentrum in Kulturbereich der orthodoxen Kirche gelegen haben. Für die Zeit, in der es wirksam gewesen sein muss, haben wir ein ausgezeichnetes Bestimmungsmittel: den vollständig gleichartigen und doch äusserst eigenartigen Jägerkalendertyp in zwei so weit voneinander entfernten Gebieten: Nordrussland und Ostsibirien. Er kann nicht in den letzten Jahrhunderten dahin gelangt sein, weil sonst ron seinem Weg noch Spuren zu finden wären. Ausserdem stellt er einen Spezialfall dar, die Anpassung des Stabkalenders an die Ausrüstung des Jägers in den eigentlichen Waldgebieten, wo kein Hausrat mitgenommen wird, weshalb das kleinstmögliche Format gewählt werden musste. Es liegt nahe, die waldigen Jagdgefilde auf der Westseite des mittleren und nördlichen Lral als sein Ursprungsland anzunehmen. Vom Anfangspunkt seiner Entwicklung bis zu sciner Verbreitung nach Ostsibirien verging sicherlich geraume Zeit. Wir können wohl mit einiger Wahr- 
scheinlichkeit schätzen, dass der Jägerkalender spätestens zu Anfang der Kolonisierung Sibiriens in (tebrauch kam. Ausserdem sind die Stabkalender, die auch bis nach Sibirien gelangten, vor allem wohl durch die Altgläubigen, bekannt und in Brauch grewesen. Damit kämen wir für den terminus ad ruem der Banernkalender in diesem Kulturbereich ins 15 . oder auch 14. (?) Jahrhundert. Wo lag nun das gemeinsame Zentrum, von dem aus die Kerbkalender sich an die Peripherie des christlichorthodoxen Kulturbereichs verbreiteten?

Da uns von Kerbkalendern im eigentlichen Russland so gut wie nichts bekannt ist, müssen wir uns in der Nachbarschaft umsehen. In Polen scheint es Stabkalender noch bis Ende des vorigen Jahrhunderts gegeben zu haben. Die Nachrichten darüber ${ }^{1}$ sind aber zu unbestimmt, als dass wir daraus Vergleichsmaterial gewinnen könnten.

Dagegen ist der Blick nach dem Balkan sehr lohnend. Hier ist es ror allem Bulgarien, wo Stabkalender erhalten geblieben sind, deren Ähnlichkeit mit den oben beschriebenen Bauernkalendern überrascht. Die erste wichtige Veröffentlichung über bulgarische Kerbkalender (raboš) stammt von S. ARGirov². Der von ihm beschriebene Kalender aus Trrnovo-Sejmen ist ein schmaler IIolzstab von 1,315 m Länge, auf dessen einer Seite die Monate März - August, auf der anderen Seite September Februar mit ilıren Tagen eingekerbt sind. Die einzelnen Ionate sind durch Zwischenräume deutlich voneinander getrennt. Ich kann in diesem Zusammenhang nicht auf alle Einzelheiten des Kalenders ${ }^{3}$ eingehen, möchte aber erwähnen, dass die kleinen Irriecke (Vrrtiefungen unter den Kerbstrichen, siehe Abb. 5a) die Sonntage begeichnen. Auf (irund deren

1 Siehe K. Moszy nski, Kultura ludova Slowian II. Kultura duchowa, Kraków 1939, S. 1617, wo Fig. 397 ein aus dem Gedächtnis nachgebildeter Kalenderstab abgezeichnet ist.

2 "Kalendar raboš» in: Periodičesko spisanie na Bălgarskoto Knižovno Družestvo v Średec, t. 54, 1896, S. 779-785. Der Staatsbibliothek in Sofia sage ich für freundliche Übersendung einer Fotokopie des Artikels aufrichtigen Itank.

${ }^{3}$ Herr Dozent Dr. Pollok (Göttingen) hat sich freundlicherweise nach dem Original erkundigt, aber nur erfahren können, dass es im Kírieg verlorengegangen ist. 


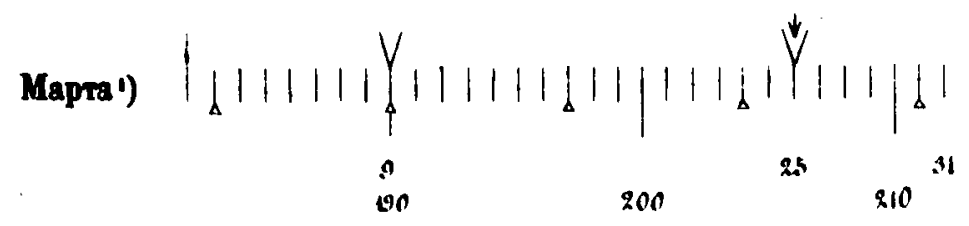

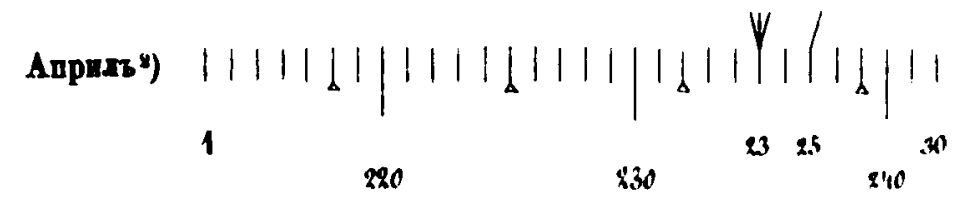

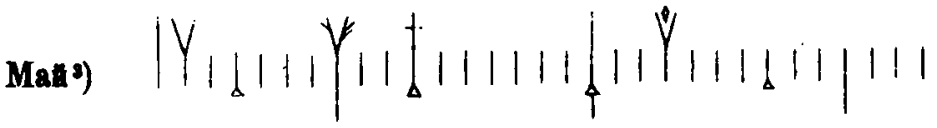

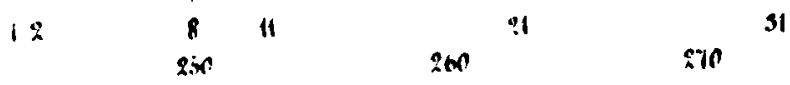

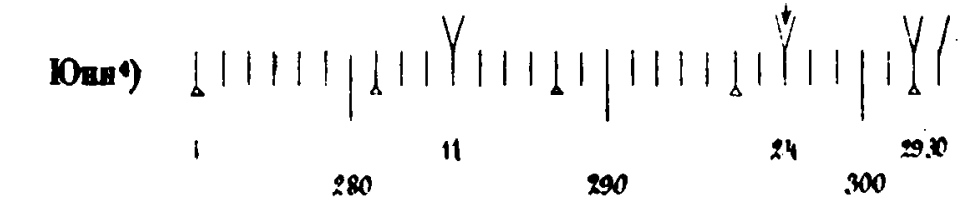

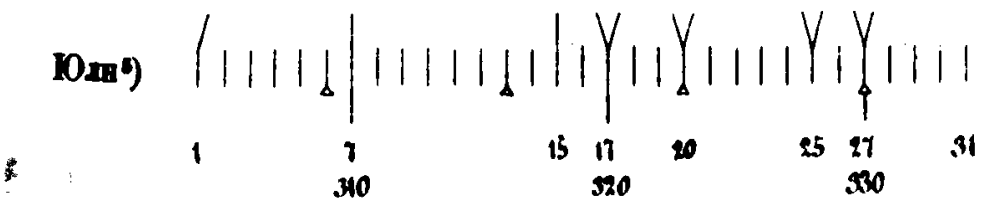

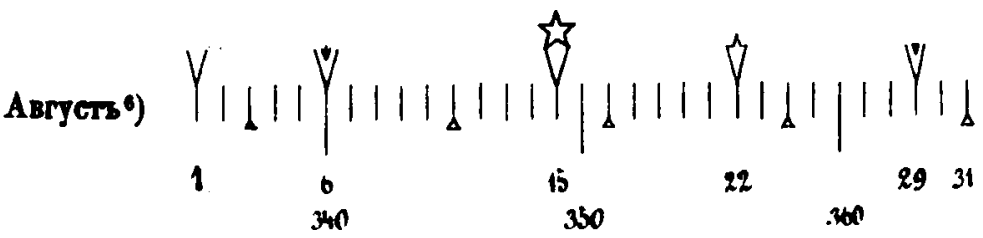

Abb. 5 Bulgarischer Kerbkalender (rabos): a) Schema der Kalenderrechnung nach $\mathrm{S}$. Argirov in: Periodičesko spisanie na Bălgarskoto

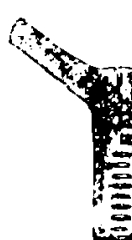




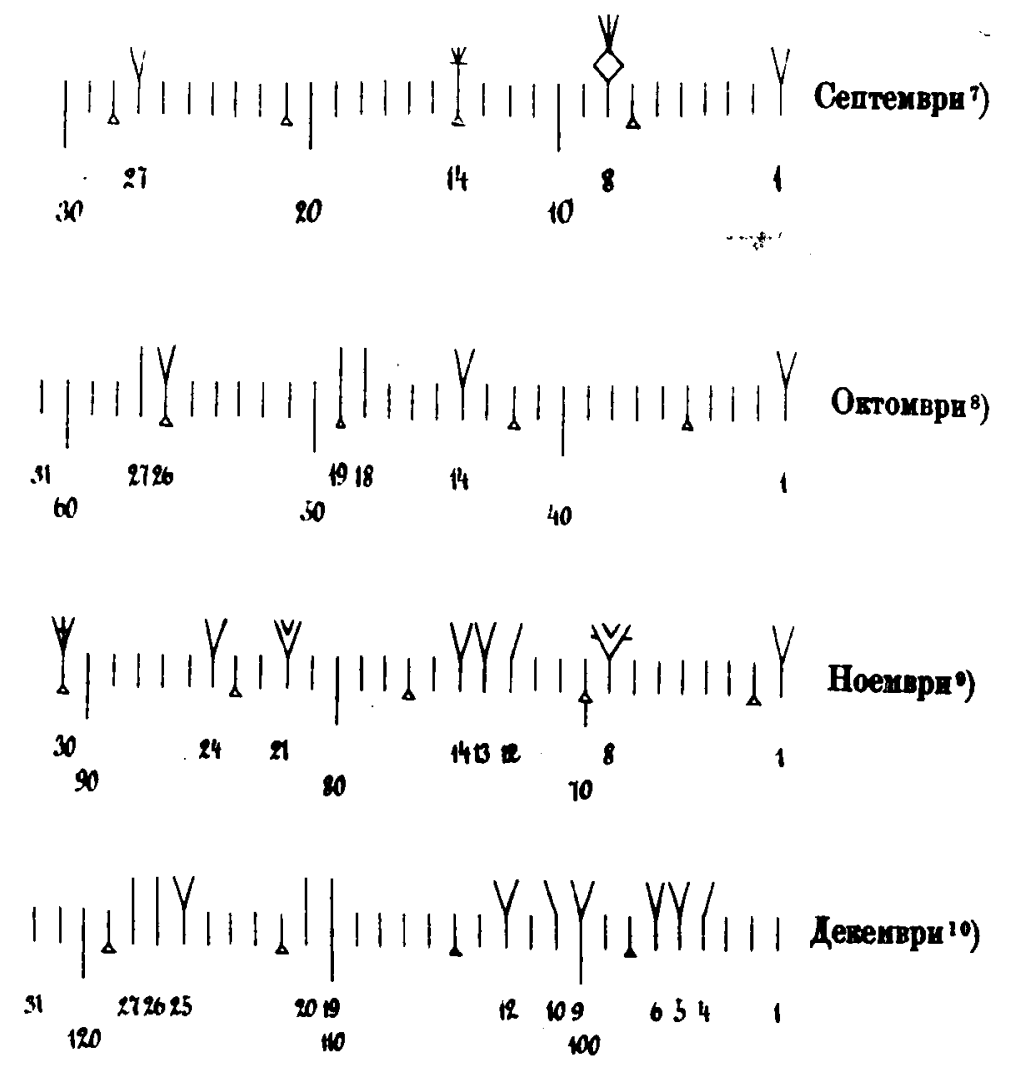

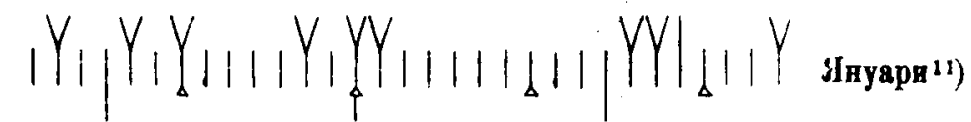
$\begin{array}{llllll}3130 & 27 & 25 & 20 & 18 n & 1 \\ 150 & & & 140 & 130 & 1\end{array}$

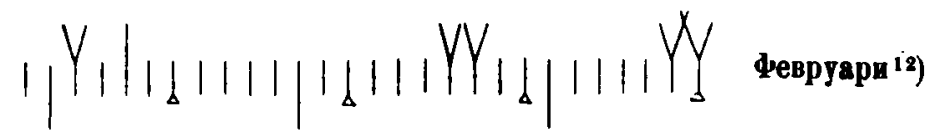
$\begin{array}{ccccc}28 \text { 2n } 21 & 1110 & & 100 & 11\end{array}$

Knižovno Družestvo $v$ Šredec, t. 54, 1896, S. 779 ff. b) Kalenderstab nach D. Marinov in: Sbornik za narodni umotvorenija i narodpis XXVIII, Tafel V. 
berechmete $\mathrm{s}$. Argirov als das Herstellungsjahr des Kalenders 1852. Gleichzeitig weist er nach, dass der Jahresbeginn anf den 1. März zu legen ist, nicht auf den 1. September, wie man nach einigen Anzeichen und dem Kirchenjahr alten Stils im damaligen Bulgarien vermuten möchte. Die Monate Januar - Februar liegen nach der Rechnung Argirovs bereits i.J. 1853. In diesem auf den ersten Blick überraschenden Jahresanfang mit Järz sieht Argirov mit Recht einen älteren Brauch, den er übrigens noch in einem bulgarischen Jahreskalender von 1842 bezeugt findet.

Diese Kalenderrechnung und vor allem die IIonatseinteilung habe ich ausführlicher gebracht, weil darin die auffallende İbereinstimmung mit den syrjänischen Kerbkalendern zu sehen ist. In den Hauptzügen: Einteilung des Jahres in IIonate, Jahresanfang mit März, Festtage des orthodoxen kirchlichen Kalenders, Zahl der Jahrestage passt der bulgarische Kerbkalender genau zı den ș̣jänischen Jägerkalendern. Mit dem syrjänischen Baluernkalender stimmt er ausserdem sogar in der Stabform und - was das Auffallendste ist - in der Art der Zeichen: den einfachen Linien und Winkeln überein. Dadurch wird der Bauernkalender aus Vizinga, der in der Kalenderforschung bisher nicht beachtet wurde, zu cinem wichtigen Verbindungsglied zwischen den Kerbkalendern des Balkans und Nordrusslands.

Der von Argirov beschriebene bulgarische Kalender ist nicht der einzige aus Bulgarien bekannte. Nach den Darstellungen von D. Marixov ${ }^{1}$ sind Kerbkalender nicht nur in der Umgebung von Haskovo, sondern auch in Čirpan und Staro-Zagora, also in südlichen Teilen des mittleren Bulgariens gefunden worden. Aus der Fotokopie, die Marinor glücklicherweise veröffentlicht hat ${ }^{2}$ (siehe Abb. 5b) und aus seinen Erläuterungen (op.cit. S. 270) geht hervor, dass die Grundlage der Kalenderrechnung die Monatseinteilung mit März als Jahresanfang bildet. Die dreieckförmige Vertiefung unter der Kerbe des 2. März ist nach dem Forscher der Schlüssel für die Berechnung der Wochentage.

1 Izvestija na Etnografičeski muzej I, Sofia 1907, S. 11 und Sbornik za narodni umotvorenija i narodpis XXVIII, Sofia 1914, S. 269 ff.

2 Sbornik za narodni umotvorenija XXVIII, Tafel V. 
Der auf dieses llatum fallende Wochentag wiederholt sich an jedem 7 . danach folgenden 'Tag. (Dieses Zeichen spielt also eine ähnliche Rolle wie der "Sonntagsbuchstabe» der skandinavischen Runenkalender.)

Noch ein dritter Beitrag zu den in Bulgarien gefundenen Kalendern ist zu erwähnen. Jor. ZAHARIEv' berichtet von einem Fund im westlichen Bulgarien in einem abgelegenen Bergwinkel der Umgebung von Kjustendil. Aus seinen Zeichnungen zur Darstellung der Kalenderrechnung und den Fotokopien des Stabes ist ersichtlich, dass dessen Markierung noch einfacher ist als die der vorhergenannten. (Die "Zugaben" der Wochenrechnung z.B. fallen weg.)

Noch weiter nach Westen führen uns die Kalenderfunde in Jugoslawien. Ther diese informieren uns am besten die Forscher Mil. S. Filipović und Milovan Gavazzi². Die von ihnen erwähnten ältesten Berichte über einen Holzkalender ${ }^{3}$, der nach Filipović aus der Hercegowina stammt, bestehen leider nur aus kurzen Notizen, so dass sich über die Kalenderrechnung nicht viel sagen lässt. Interessant ist der Bericht von M. S. Filipović ${ }^{4}$, dass er noch 1956 an den IIöhen des Treskavica im Gebiet von Sarajevo alte I,ente traf, die sich an den Gebrauch von Kerbkalendern um die Jahrhundertwende noch erinnern konnten. Glücklicherweise konnten an der nördlichen Küste Dalmatiens auf der Insel Olib Kerbkalender gefunden werden, über die M. Gavazzi berichtet und Fotokopien veröffentlicht hat. Einer dieser Kalender von Olib ${ }^{5}$ ist ein vierkantiger $4 \mathrm{~cm}$

1 Izvestija na Narodnija etnografski muzei v Sofija VIII-IX, Sofia 1929, S. $242-248$ mit 4 Abb.

2 An dieser stelle möchte ich Herrn Prof. Alois Schmaus (München) für die Vermittlung ihrer Anschriften wie auch den Gelehrten selbst für die liebenswürdigen Auskünfte und die I"bersendung von Separata meinen herzlichen Dank aussprechen.

3 Glasnik zemaljskog muzeja u Bosni i Hercegovini III, Sarajevo 1891, S. 457-458 und Zbornik za narodni život i običaje južnih Slavena, I, Zagreb 1896, S. 41 .

- Raboš-kalendar u okolini Sarajeva. Glasnik Zemaljskog muzeja u Sarajeva, N. S. XIII.

5 M. Gavazzi, Drveni kalendar - rovaš sa Jadrana, Narodna starina I X, Zagreb 1930, S. 331-333. 
breiter und 74 cm langer Holzstab, auf dessen Seiten jeweils drei Monate mit ihren Tagen und Festen eingekerbt sind (siehe Abb. 6). Diesem ganz ähnlich ist ein zweiter Kerbkalender ron Olib über dessen Kalenderrechnung Gavazzi ${ }^{1}$ ein Schema vorlegt $^{2}$ (Abh. 7). Wie man sipht, giht es darauf keine Wochenrechnung. Der Unterschied zu den hulgarischen Killendern besteht vor allem im Jahresanfang, dem 1. Januar, und in den römisch-katholischen z.'T. rein lokalen Festen. Wir haben es hier wohl mit einer Anpassung des bulgarischen Kalendertyps an die Kalenderrechnung der römisch-katholischen Kirche zu tun. In diesem Sinne glaube ich din Schlussbemerkung im Artikel M. Gavazzis in Narodna starina IX S. 333 verstehen zll dürfen, wo er sagt, dass dieser Holzkalendertyp nach der Insel Olib seinen Wer durch den Balkan in der Richtung nach Nordwesten genommen hat und mit dem bulgarischen Typ im wesentlichen übereinstimmt.

Auch aus anderen Teilen des Balkan liegen Nachrichten ïber Kerbkalender vor. Aus Albanien sind einige ca. $1 \mathrm{~m}$ lange Stäbe "mit runenartigen Einkritzungen, die als Kalendarien dienten" in das ethnographische Museum in Genf gelangt. ${ }^{3}$ íber Kalenderstäbe bei den Slowenen berichtete schon A. MǗlLNer in "Argo", Zschr. für krainische Landeskunde, und neuerdings u.a. Vilfas ${ }^{4}$. Bis ins Banat führt uns schliesslich Mil. S. Filipoviós. In Tngarn sind keine Kerbkalender mehr vorhanden. ${ }^{6}$

Es würde zu weit führen, auf die interessanten Stabkalender

1 "Drveni kalendari" in Kalendar Sv. Ante za g. 1939, Sarajevo 1938.

2 Ijiese Kalender wurden, wie Gavazzi sagt, von den Bauern gewöhnlich nach einem alten Muster hergestellt. Sie konnten an dem einen durchbohrten Ende an einer Sclnur aufgehängt werden. Sie waren in Gebrauch bei den Hirten, die fern von den Siedlungen in den Bergen bei ihrer Herde weilen.

3 L. Rütimeyer, Ur-Ethnographie der Schweiz, S. 36.

4 Sergij Vilfan, "Očrt slovenskega pravnega narodopisja" in: Narodopisje slovencev I, Ljubljana 1944, mit Abb. S. 246.

5 Raboš-kalendar u Banatu. Zbornik Matice Srpske. Serija Društvenih nauka 2, Novi Sad 1951, S. 121 ff. Diese Schrift war mir leider nicht zugänglich.

- Gyula Sehest yén, Inovis es rovásiris, ‥ 100 . 
Abb. 6 Kerbkalender (ragoš) von der Dalmatischen Insel Olib.

Foto: M. Gavazi in: Narodna starina, IX, 7agreb 1930, S. 333.

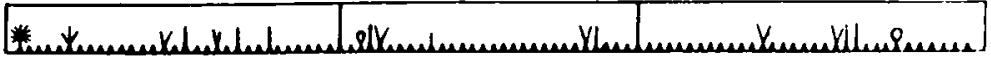

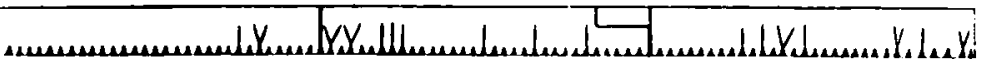

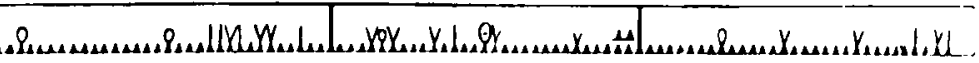

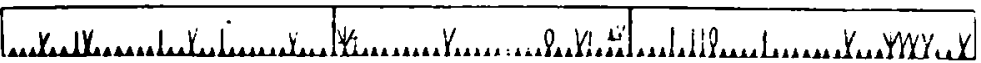

Abb. 7 Schema der Kalenderrechnung eines Kerbkalenders von Olib nach M. Gavazzi in "Kalendar Sv. Ante", Zagreb 1939.

aus den Österreichischen Alpen einzugehen. Ich kann hier nur summarisch auf die Untersuchungen des mit diesem Thema besonders vertrauten Forschers Robert ScIINDLER ${ }^{1}$ und auf einen Artikel von G. GraBer ${ }^{2}$ hinwcisen. In diesem werden zwei Stabkalender aus Kärnten (aus der Mosinz nordöstlich von Hüttenberg) genau beschrieben. Der jüngere von ihnen stammt aus d.J 1685. Sie haben die Form eines Miniatur-Schwertes mit Griff, voll dem her dir Striche und Zeichen für die 365 .Jahrestage, die, jewrils in 7 Tage eingeteilt sind, gezählt werden. Die Zählung beginnt mit dem Neujahrsfest. Die Monatsanfänge sind nicht

1 "Die österreichischen Volkskalender", St. Josephs-Kalender 1949 , S. 103; "Die Holzkalender in Österreich und Frankreich", Beiblatt zu "Österreichische Furche", 1. Jan. 1949 u.a.m. Für seine liebenswürdigen Auskünfte, Literaturhinweise und Separata sage ich ihm herzlichen Lank.

2 Holzstabkalender in Kärnten. Festschrift für R. Egger. (Beiträge zur älteren europäischen Kulturgeschichte III.) Klagenfurt 1954. 3 Abb. S. $372 \mathrm{ff}$. 
bezeichnet. In Form und Zeitrechnung wie auch in den durch symbolische Figuren anschaulich gemachten Frst- und redenktagen sind diese Stäbe nach Graber den schwedischen Runenstäben überaus ähnlich, so dass der Verfasser an deren Herkunft aus Schweden denkt! Für unsere Untrrsuchung ist die Feststellung ron Wichtigkeit, dass die Alpen offensichtlich eine Grenze sind, wo die östlichen und die westlichen Kerbkalenderstäbe sich treffen. Um also auf unserem Gebiet, dem der orthodoxen Kirche, zu bleiben, kehren wir nach Bulgarien zurück.

Hier erleben wir noch eine Überraschung. Zn den Bulgaren jenseits der südöstlichen Grenze des Landes in das östliche 'Thrakien führt uns Hr. VAKARELSKI in seiner wertvollen Untersuchung1. Er berichtet, dass in der Umgebung von Samokovo (in den Dörfern Jana und Ciknihor) noch zu Anfang der 30er Jahre ds. Jhs. Kerbkalender in Gebrauch waren. Der von ihm beschriebene und mit einem Schema der Kalenderrechnung dargestellte (vielleicht um 1860 hergestellte) Kerbkalender ist ein $117 \mathrm{~cm}$ langer vierkantiger Stab mit der Einteilung des Jahres in Monate, die zu je 6 auf zwei Flächen mit ihren Tagen (30 bzw. 31, für Febr. 28) durch ähnliche Kerben markiert sind wie die von Arorirov und Marinov behandelten. (Die Verteilung der Monate Oktober - März, April - September ist ein merkwürdiger Zug.)

Das Eigenartigste, was wir hier erfahren, ist die Mitteilung über den Ursprung des Kalenders. Er ist, wie Vakarelski zu berichten weiss, ins Dorf Ciknihor als Geschenk vom "Heiligen Berg» Athos mitgebracht worden. Der Forscher fügt die aufschlussreichr Bemerkung hinzu, dass derartige Kalender ganz, allgemein aus Klöstern kamen, ron Mönchen hergestellt waren und unter den Geistlichen, Psalmensängern und dem Bauernrolk verbreitet wurden. Das gilt nach dem Verfasser z.B. auch von den Kalendern aus der Umgebung von Haskovo, die Marinov beschrieben hat, und das ist u.a. auch aus den kirchlichen Festtagen zu sehen, die dem Volk fremd waren. Der Mitteilung über die Herstellung von Kerbkalendern durch Mönche auf dem

1 Bitъ i ezikъ na trakijskitě i maloazijskitě błlgari, t. 1, Sofia 1935 , S. $269 \mathrm{ff}$. 
Berg Athos und in anderen Klöstern kommt noch dadurch eine besondere Bedeutung zu, dass nicht bloss slawische, sondern auch g r i e c h i s c h e Mönche, wie Vakarelski am Ende seiner Ausführungen betont, derartige Kerbkalender hatten und verschenkten. (Die ron diesen hergestellten haben einige besondere Züge). Wir sind somit auf der Suche nach dem Zentrum, von dem die christlich-orthodoxe Kalenderrechnung, die an den syrjänischen Kerbkalendern zu sehen ist, ausgegangen sein kann, in den byzant in is chen Kulturbere i ch gelangt.

Es ist nicht schwer, sich den Weg vorzustellen, den die Kerbkalender mit der christlichen Zeitrechnung aus Griechenland und Bulgarien nach dem äussersten Norden Russlands genommen haben, wenn wir die Tatsache, dass sie in Klöstern gemacht wurden, nicht auf den Athos und Bulgarien beschränken. Auch in den Klöstern der römisch-katholischen Kirche waren sie bekannt und wohl auch in (rebrauch. Eine Handschrift des Klosters St. Gallen erwähnt schon um das Jahr 900 Kerbstöcke, die als Kalender dienten. ${ }^{1}$ Welche grosse Bedeutung die russisch-orthodoxen Klöster besonders zur Zeit der Christianisierung der finnisch-ugrischen Völker in Mittel- und Nordrussland hatten, braucht nicht eigens ausgeführt zu werden. Dass auch Verbindungen mit dem Athos bestanden, ist wohl nicht zu bezweifeln. (Ein griechischer Mönch vom Athos ist sogar bis zum Ladoga gekommen.) Es scheint also ganz natïrlich, dass von Byzanz her neben anderen Traditionen auch die Kalender in die russischen Klöster des Nordens weitergetragen und von diesen dem Volk gegeben wurden.

Welche Zeit dafür in Frage kam, ist schwer zu sagen. Wir haben oben als Anfangszeit der Verbreitung der Bauernkalender in Russland das 15 . oder 14 . Jh. angenommen. Wenn man bedenkt, dass der Bischof Stephan, ehe er seine Missionstätigkeit unter den Syrjänen in der 2. Hälfte des 14. Jhs. begann, als Mönch im Kloster von Rostov lebte, erscheint es als durchaus möglich, dass er dort die Kerbkalender (Bauernkalender) kennen gelernt und zu den Syrjänen gebracht hat. (千. S. Lrtkis ${ }^{2}$ datiert

1 Zitiert nach R. Schindler, Die österreichischen Holzkalender, S. 103.

2 Зырянскій край при епископахъ пермскихъ и Зырянскій языкъ. Санктпетербургъ, 1889, S. 24 (Tafel). 
den ron Sarvaitor beschriebenen Kalender ins 14. Jh., weil er die Festtage in genauer Übereinstimmung mit einem kirchlichen Kalender des 13. Jahrhunderts findet. Beweisen lässt sich ein so hohes Alter der syrjänischen Kerbkalender wohl kaum, da die uns erhaltenen Stücke einschliesslich des Savvaitorschen, soviel ich weiss, sich nicht in eine frühere Zeit als die erste Hälfte des 19. Jhs. datieren lassen. Unwahrscheinlich ist, dass schon zur Zeit der Christianisierung der Syrjänen der Jägerkalendertyp aufgekommen sei. Dieser unterscheidet sich in seiner Form und vor allem in der Graphik (den Eigentumszeichen und Figuren, die kaum eine Beziehung zum Balkan laben) so sehr von dem bulgarischen Banernkalendertyp, dass wir eine Entwicklung aus cinheimischen Kulturelementen annehmen müssen.

Die Anwendung von Eigentums- bzw. Familienzeichen (Pas) zur Kennzeichnung der Fest- und redenktage dürfte sich meines Erachtens aus dem sehr alten Brauch der Permier, Kerbhölzer mit Eigentumszeichen zu versehen, entwickelt haben. Einen interessanten Beleg für das letztere bringt Uno HARVA in "Permalaisten uskonto» $\mathrm{S} .109$ für die Wotjaken. Die Teilnahme an den Opfern im Lud wird in der Weise maufgezeichnet", dass auf der schmalen Seite eines vierkantigen Holzstäbchens die Geldsumme der einzelnen Spender in römischen Buchstaben und auf der darïberliegenden breiteren Fläche über der jeweiligen Zahl das Eigentumszeichen der Familie oder der Personen des betr. Hauses eingekerbt werden. Die Ähnlichkeit einer solchen "Zahlen- und T'eilnehmerliste", die Harva auch in einer Zeichnung (ib. Abb. 28) darstellt, mit dem Zählund Zeichensystem der Jägerkalender ist so deutlich zu sehen, dass man an einer Ủbernahme des graphischen systems kaum zweifeln kann. Man darf wohl auch annehmen, dass die PasZeichen der Jägrerkalender - wenigstens im Anfangsstadium der Übernahme - ihre ursprüngliche Bedeutung noch besassen. Darauf deutet auch die Tatsache, dass die Zeichen für die bestimmten Fest- und Gedenktage (wie auch deren Zahl selbst) sehr verschiedenartig sein können (vgl. die beiden Jägerkalender Abb. 1) und nicht, wie Savvaitov auf Grund eines einzigen Kalenders meinte, feststehende symbole für 
die betr. Feste sind. Die Einführung derartiger Zeichen in den christl. Kalender ist als eine eigene Leistung der Permier zu betrachten, vergleichbar der Einführung der Runen in die nordische Kalenderrechnung durch die Skandinavier.

Ausserdem bedeutet unsere Feststellung, dass das Zeitrechnungssystem der syrjän. Kerbkalender byzantinischen Ursprungs ist, keineswegs, dass die Syrjänen, als sie es durch Vermittlung der Russen übernahmen, keine eigene Zeitrechnung gehabt hätten. Im Gegenteil. Es lassen sich Belege für ihre alte Zeitrechnung anführen. Alle diese Fragen sind noch offen und bedürfen einer rigrnen Untersuchuug.

ir. J. STIPA 